\title{
Supporting continuity in teaching French from secondary school to the university degree
}

\author{
[Pour favoriser la continuité de l'enseignement du français du \\ secondaire au supérieur]
}

\author{
Iveta Rizekova
}

DOI: $10.18355 / X L .2018 .11 .01 X L .13$

\begin{abstract}
Based on the fact that the level of French competence among the students applying for university education has been decreasing, the main idea of this article is to draw attention to the importance of continuity in French learning between secondary school and university within the context of Slovak education. Continuity is a very important precondition for effective learning of languages, as it enables the learners to build on their previous language competence and create conditions for future development of language knowledge and skills as well as communication and cross-disciplinary competencies. Based on relevant differences between secondary and tertiary environment we will point out some difficulties, the students may face at tertiary level. Simultaneously, we will recommend some strategies and practices to overcome them and ensure a harmonious passage between secondary and tertiary level of language education.
\end{abstract}

Key words: continuity, French language, competencies, university, secondary school

\begin{abstract}
Résumé
$\mathrm{Vu}$ le fait que le niveau de français des candidats aux études universitaires baisse, la présente communication se donne pour objectif principal de porter l'attention sur l'importance de la continuité dans l'apprentissage du français dans le contexte de la formation slovaque, dès l'école secondaire jusqu'à l'université. La démarche continue est une condition nécessaire à l'apprentissage efficace d'une langue, comme elle permet de nouer aux compétences précédentes et de prévoir le futur développement des savoirs et savoir-faire langagiers, ainsi que celui des compétences communicatives et transversales. A la base des différences pertinentes entre le milieu secondaire et universitaire, nous tâchons de signaler certaines difficultés que les étudiants peuvent rencontrer à l'université. En même temps, nous proposons des stratégies et démarches permettant de les dépasser et de rendre la transition entre le cycle secondaire et universitaire de la formation linguistique harmonieuse.

Mots-clés: continuité, langue française, compétences, université, école secondaire
\end{abstract}

\section{Introduction}

Dans le monde globalisé, la maîtrise des langues étrangères est un excellent moyen de multiplier les opportunités professionnelles pour les jeunes, en leur permettant d'accéder aux emplois où la communication en langue étrangère est une nécessité ou un avantage. Les jeunes en Slovaquie se rendent de plus en plus compte que pour réussir leur carrière, parler une langue étrangère est un atout majeur.

La Slovaquie, comme un pays membre de l'Union européenne, doit répondre aux nouveaux défis de l'enseignement, c'est-à-dire favoriser l'autonomie de l'apprenant et la prise en charge de son apprentissage tout au long de ses études. On attend de l'apprenant qu'il sache coopérer aussi bien avec les autres apprenants qu'avec l'enseignant afin de définir les objectifs à atteindre et la méthode à adopter, afin d'apprendre à contrôler et à évaluer sa progression, et de partager ses savoirs et ses expériences (Camillieri, 2002 : 5). Cette approche pédagogique permet de conforter sa 
motivation et confiance en soi. L'enseignant cesse d'être pour lui une autorité infaillible et devient plutôt un facilitateur de son apprentissage. Un bon pédagogue comprend les besoins personnels de ses apprenants et mobilise leurs propres capacités à réaliser des activités diversifiées. Evidemment, le progrès dans l'apprentissage apporte le plaisir d'apprendre qui présente un gage de réussite.

Il est à rappeler que ce contexte d'enseignement/d'apprentissage est indissociable de la notion de compétence. Marc Romainville définit celle-ci comme un ensemble intégré et fonctionnel de savoirs, savoir-faire, savoir-être et savoir-devenir permettant s'adapter à des situations données, de résoudre des problèmes ou encore de réaliser des projets (Houchot - Robin, 2007: 12). En effet, l'apprentissage d'une langue devrait conduire non seulement aux savoirs, mais surtout au développement des capacités cognitives et des attitudes socio-affectives. D'où l'attachement de la compétence au contexte social défini. D'après Tilman « être reconnu compétent, c'est aussi appartenir à un groupe et s'inscrire dans une vie collective...» (Tilman, 2006). C'est pourquoi l'enseignement basé sur le développement des compétences devrait sensibiliser les apprenants à observer le milieu où l'acte de communication se déroule et à ajuster leurs énoncés à la situation donnée.

Si l'apprentissage d'une langue étrangère est l'affaire d'un processus continu, l'enseignement du français devrait suivre un processus identique, et cela à partir du degré secondaire (le cas échéant du primaire) jusqu'au supérieur. Dans le souci d'assurer une démarche cohérente, il faut créer des rapports harmonieux entre ces cycles d'apprentissage, et cela en matière d'identification des besoins, de détermination des objectifs et des contenus d'enseignement, d'adaptation des méthodes pédagogiques et

de la mise en œuvre des mécanismes de contrôle et d'évaluation équitables. Il est évident que toute université tâche de pousser les apprentissages plus loin et à un niveau plus élevé, même si leurs formes d'organisations peuvent varier selon les pays ou d'un établissement à l'autre.

Bien que la formation scientifique de qualité reste la première priorité de l'enseignement universitaire, il n'empêche que les établissements d'éducation postsecondaire doivent tenir compte des mouvements démographiques, politiques et socio-économiques dans la société dans le but de favoriser l'insertion de leurs diplômés au marché du travail. Afin de promouvoir une meilleure intégration au marché du travail dans le contexte national, voire international, les profils de sortie des diplômés priorisent, à part des savoirs disciplinaires, des compétences en langues. Les métamorphoses dans le milieu universitaire slovaque se traduisent non seulement au niveau des effectifs d'étudiants, mais aussi au niveau de variété de curricula. La possibilité de choisir parmi une pluralité de programmes ou de spécialités, ainsi que parmi les niveaux d'accès et niveaux de langues différents est aujourd'hui accordée à un plus grand nombre de bacheliers. Les filières d'études sont plus perméables qu'auparavant, ce qui favorise les passerelles d'une formation à l'autre. De plus, cet enseignement passe du monolingue au plurilingue et pluriculturel, en raison de la possibilité de faire une période d'études à l'étranger grâce à la mobilité étudiante. Néanmoins, nous constatons que tous ces privilèges n'entraînent pas obligatoirement que des effets positifs. Le pire est que les ressources financières consacrées au fonctionnement des universités et au développement de la recherche sont assez restreintes. C'est ainsi que chaque établissement cherche d'autres moyens, hormis ceux fournis par l'Etat, pour subventionner ses travaux de recherche, ses parcs technologiques ou encore pour nouer des partenariats avec des entreprises en vue de s'insérer davantage dans le paysage local.

Bien qu'il y ait de plus en plus d'étudiants travaillant à temps partiel, en parallèle de leurs études supérieures, un bon nombre de diplômés éprouve des difficultés à s'insérer sur le marché du travail à la fin de chaque année académique. Ils se posent 
donc des questions : Comment remédier à cette situation qui est loin d'être de courte durée ? Comment équilibrer l'acquisition des connaissances et leur exploitation dans l'apprentissage? Il ne sera pas facile à trouver un seul remède à ces problèmes qui ont un caractère multidimensionnel et impliquent tous les acteurs du secteur de l'enseignement national.

\section{La présence française en Slovaquie}

Avant de traiter la problématique de l'enseignement du français actuel dans les cursus scolaires slovaques, il nous semble pertinent de la mettre en contexte des circonstances sociales, économiques, politiques et éducatives importantes des dernières années. Après l'adhésion de la Slovaquie à l'Union européenne, la présence française dans le pays est devenue plus importante et la position du français comme langue de travail dans les institutions européennes a rendu cette langue encore plus attractive. Etant donné les relations étroites entre la France et la Slovaquie encadrées par un accord de partenariat stratégique, signé à Paris en 2008 et renouvelé pour les années 2013-2018, dont le programme de coopération comprend cinq volets : politique, économique, militaire, énergétique et administratif, l'enseignement du français a pris de l'importance (Relations bilatérales).

Actuellement, "la France est le $6^{e}$ partenaire commercial et le $11^{e}$ investisseur en Slovaquie (884 millions $€$ de stock, représentant 400 entreprises employant 30000 personnes) » (Relations bilatérales). Les entreprises du secteur d'industrie automobile, de construction, des services financiers (assurances, services de crédit et bancaires), de télécommunications, de l'énergie, la chimie, la pharmacie et des services, sont bien positionnées en Slovaquie. "Les autorités slovaques souhaitent attirer les capitaux français, en particulier dans certains secteurs prioritaires : infrastructures routières, énergie - en particulier nucléaire, numérique, défense " (ibid.). La Chambre de Commerce Franco-Slovaque (CCFS), créée en 1994, a pour objectif « de développer le commerce entre la France et la République Slovaque, de favoriser le développement des relations bilatérales entre ces pays et d'aider à l'augmentation des investissements français en Slovaquie. ... En juin 2011, la CCFS a signé le contrat de partenariat avec UBIFRANCE, l'Agence pour le développement international des entreprises et est ainsi devenue le seul interlocuteur pour accompagner les entreprises françaises dans leur développement en Slovaquie » (Historique et objectifs).

Le nouveau plan d'action mentionné ci-dessus a pour but «d'intensifier le dialogue, de développer les liens économiques (investissements croisés, échanges commerciaux, flux touristiques) et de concrétiser les coopérations culturelles, en particulier dans le domaine éducatif » (Relations bilatérales).

\section{La position du français en contexte scolaire slovaque}

La coopération linguistique et éducative entre la Slovaquie et la France s'appuie sur un réseau comprenant l'Institut français de Bratislava, deux Alliances françaises, quatre sections bilingues franco-slovaques, récemment labellisées «France Éducation ", l'École française de Bratislava qui proposera à terme une double certification baccalauréat français/maturita slovaque. Un arrangement relatif à la coopération éducative, linguistique, universitaire et scientifique pour la période 20162019 a été signé en novembre 2016 à Paris. Depuis 2002, la Slovaquie a le statut de membre observateur de l'Organisation internationale de la Francophonie (Relations bilatérales).

La coopération scientifique et universitaire est axée sur la poursuite d'études en France, les collaborations universitaires franco-slovaques sur le plan académique et de recherche. La Slovaquie compte aujourd'hui 18 programmes en français ou à la française, dont 5 programmes qui peuvent mener à une double diplomation. Ils permettent d'effectuer des parcours universitaires francophones allant jusqu'au 
diplôme de master. En plus, les étudiants ont la possibilité d'effectuer une partie de leur cursus en France : il existe environ 30 conventions bilatérales et 160 conventions Erasmus entre universités françaises et slovaques. Chaque année, environ 500 étudiants slovaques se rendent en France pour y suivre au moins un semestre d'étude. Par contre, une centaine d'étudiants français viennent passer 6 mois ou plus en Slovaquie. Les bourses sont généralement attribuées à des étudiants slovaques allant faire une deuxième année de Master (M2) et aux thèses faites en cotutelle francoslovaque (ibid.).

L'espace commun européen a relevé en Slovaquie la nécessité réelle de savoir communiquer en langues étrangères. D'après le Programme national d'éducation et de formation en vigueur, la maîtrise des langues étrangères constitue un des piliers de la politique linguistique slovaque : "Il semble nécessaire d'introduire le principe d'un parcours continu de l'enseignement des langues (au passage de l'Enseignement fondamental à l'Enseignement secondaire) dans tous les programmes des différentes écoles secondaires, dans le but de développer la capacité des apprenants à communiquer en deux langues étrangères » (Profil, 2004-2007 : 60). Dans le Profil de politique linguistique éducative, quatre zones de transversalité demandent considération attentive et font l'objet de propositions pour l'avenir.

- $\quad$ «la continuité / discontinuité dans les cursus et entre les niveaux,

- la formation des enseignants,

- l'avenir des départements de langues des universités,

- l'évaluation et la certification » (Profil, 2004-2007 : 54).

D'une part, la pénétration des investisseurs en Slovaquie crée un climat favorable à l'élargissement du français et de son apprentissage. En effet, l'opportunité de trouver un emploi dans une entreprise ou une société française, après la fin des études, s'avère être une forte motivation. D'autre part, en raison de la forte présence de l'anglais dans la société slovaque, plusieurs entreprises favorisent la communication en anglais. Cependant la maîtrise du français reste un grand avantage. Il est certain que l'anglais s'affirme dans le monde professionnel comme la langue véhiculaire, et devient pour cette raison la première langue choisie par les écoliers. De même, dans tous les types d'écoles en Slovaquie, "c'est l'anglais qui est la langue la plus fréquemment enseignée, suivie de l'allemand, les autres langues figurant très loin derrière » (Profil, 2014-17 : 19). Au cours de la scolarité obligatoire et par suite au degré supérieur, il est toutefois possible d'apprendre le français, le russe, l'espagnol ou l'italien etc. Par contre, la possibilité de continuer à apprendre la langue dont l'apprentissage a commencé à l'école fondamentale n'a existé à $80 \%$ que pour l'anglais et l'allemand, tandis que dans le cas du français plus de $50 \%$ des élèves n'ont pas poursuivi l'étude de la langue apprise auparavant, à savoir trois quart de ceux-ci recommencent «à zéro » (ibid. : 20-21). Ce constat d'un défaut de continuité devient un facteur de préférence de l'anglais ou l'allemand, au détriment du français.

Les enseignants de français ne sont pas du tout satisfaits de la position occupée par le français et s'inquiètent à bon droit de son avenir. A savoir, à tous les niveaux d'enseignement obligatoire et aussi post-baccalauréat, les effectifs d'apprenants diminuent, ce qui se reflète dans la qualité même des connaissances langagières. En Slovaquie, la langue française occupe la position de deuxième ou troisième langue apprise. Et dans la plupart de cas, elle n'est dispensée qu'à partir du cycle secondaire, c'est-à-dire surtout aux lycées, comme dans les écoles professionnelles elle est enseignée rarement. Etant donné une régression considérable dans l'enseignement du français dans les écoles primaires et secondaires slovaques (l'anglais et l'allemand y prédominent) durant ces dernières années, et par conséquent, une baisse des inscriptions aux filières universitaires, les organismes d'enseignement supérieurs et 
les enseignants de français doivent déployer maintes activités pour attirer de nouveaux étudiants.

Nous n'avons qu'à consentir à l'idée que «La continuité entre différents degrés scolaires (continuité verticale) ne peut être effectivement assurée si une cohérence horizontale (transversale) entre les différentes matières et les différentes langues n'est pas aussi prise en compte " (Profil, 2004-2007 : 18). Malgré un grand nombre de programmes d'études en langues aux universités slovaques, y compris ceux en français, le nombre d'inscriptions va décroissant. Hormis les raisons démographiques défavorables, c'est la discontinuité du système de l'enseignement des langues, dès l'école primaire jusqu'à l'université, qui aggrave cette situation. Celle-ci découle, d'une part, de la transgression de la diversité linguistique et d'autre part, des attitudes stéréotypées dans le public : que le français est une belle langue, mais impossible à apprendre pour une prononciation et grammaire difficiles; qu'il n'est parlé qu'en France et d'autres. Après l'anglais, c'est la langue allemande qui est la plus préférée, pour des raisons historiques ainsi que pour la proximité de deux pays germanophones. Or, il est bien logique que l'absence de français au niveau primaire et secondaire engendre un manque d'apprenants au cycle supérieur. Ce qui est pire encore, c'est que les bacheliers n'ayant pas atteint le niveau exigé en français (ce qui peut être causé par un nombre de cours ou d'années d'apprentissage restreints), ont craintes de l'échec et choisissent alors une autre langue, si c'est possible. Il est à noter que la situation varie selon les conditions dans chaque université, ainsi que selon l'attitude et la qualité des enseignants. Les institutions compétentes devraient prendre en compte la gravité de cette situation et œuvrer pour promouvoir la diversité linguistique. "On peut rendre la position respectable au français en Slovaquie par les efforts concertés des enthousiastes, de l'Ambassade et des responsables des politiques linguistiques de la Slovaquie... Un contact direct, une campagne ciblée, l'abordement direct des parents, la motivation et un recyclage de qualité des enseignants slovaques de français $d u$ premier degré sont donc à l'ordre du jour » (Kopecký, 2007 : 73).

La formation des pédagogues de français présente un autre problème à relever. Aujourd'hui, l'enseignement du français est dispensé à cent pour cent par des pédagogues qualifiés, par contre son avenir se montre menacé par l'âge assez élevé de la plupart d'eux (Fanová, 2013). Il manque toujours une nouvelle génération de pédagogues de français, car la plupart des diplômés des filières pédagogiques trouve leur emploi dans les entreprises ou travaille comme interprètes, c'est-à-dire dans les métiers mieux rémunérés. En Slovaquie, la profession de pédagogue a perdu beaucoup d'attractivité au cours de dernières années.

\section{L'enseignement supérieur versus l'enseignement secondaire}

L'école secondaire et l'université représentent deux espaces d'enseignement et d'éducation qui se distinguent en plusieurs points. La première différence se manifeste au niveau de la quantité et la qualité des contenus disciplinaires. Comme l'université dispense la formation correspondant aux disciplines scientifiques ou appliquées, le côté théorique et terminologique des cours peut présenter une grave difficulté pour l'apprenant qui ne possède pas de bases solides en matières.

L'enseignement universitaire des/en langues (première ou seconde), dont l'apprentissage a débuté au cycle précédent, vise le développement et l'approfondissement du niveau de langue pour atteindre les objectifs communicatifs en rapport d'un contexte donné, c'est-à-dire à part de faire la conversation aux sujets de la vie quotidienne, les échanges et les tâches seront plutôt de type professionnel (commercial, interculturel) ou de type éducationnel (participer à un débat, écrire un article sur un sujet spécialisé) etc.

$\mathrm{Au}$ cours de dernières années, le niveau de connaissances en langues chez les étudiants inscrits aux études universitaires a une tendance descendante, ce qui les amène à la faillite ou parfois à l'abandon des études. Cet état peut être causé par 
l'inefficacité de l'enseignement secondaire qui n'intègre pas des acquis grammaticaux, lexicaux, morphologiques, syntaxiques etc. dans un contexte de communication. De l'autre côté, la faute est aux universitaires qui se font fréquemment une fausse idée sur les contenus aux écoles secondaires et ont donc la tendance à surestimer les acquis des bacheliers. Par conséquent, leurs attentes excessives peuvent produire un choc à ceux qui débutent leurs études supérieures. Finalement, il ne faut pas oublier que la réussite ou l'échec est tout d'abord l'affaire de chaque individu et dépend de sa responsabilité et sa persévérance dans les études.

Un autre problème qui surgit, c'est l'adaptation des apprenants à un nouveau milieu et nouveau système de formation, dont l'organisation de l'année académique, le déroulement des cours, la participation aux cours magistraux avec la prise de notes, le travail par projet, les activités effectuées en groupes etc. Les étudiants peuvent être désorientés par le nombre de cours et de professeurs différents. Ils ont du mal à s'adapter aux consignes de travail et d'apprentissage que donne chaque enseignant. Ils doivent s'adapter également aux nouvelles modalités d'évaluation et de certification. D'ailleurs, la communication avec les enseignants et le personnel de direction ou d'administration dans le contexte académique (et professionnel) est différente de celle avec les professeurs des écoles secondaires. Les arrivants à l'université à l'âge de 1820 ans sont traités comme des adultes chez lesquels on suppose la prise de toutes leurs responsabilités. Ces jeunes adultes sont incités à agir et à se décider de manière plus autonome. L'université, plus que le lieu de formation secondaire, exige de la part des étudiants une autonomie dans le travail et dans l'organisation. Les cours magistraux n'étant pas obligatoires exigent une forte autodiscipline de la part des étudiants. En outre, la capacité de travail de recherche incluant des exigences en rapport de méthodologie scientifique n'est pas non plus une affaire aisée. En cas de blocage qui entraîne souvent le découragement, les apprenants peuvent demander des conseils auprès de leurs professeurs, ce qui permet, en outre, d'entretenir de bonnes relations humaines.

Contrairement au monde de l'enseignement secondaire, le monde universitaire apporte de nombreuses opportunités d'échanges internationaux à travers la mobilité étudiante en Europe, notamment par le projet Erasmus+. Les mobilités sont anciennes dans les universités européennes, mais elles connaissent depuis une vingtaine d'années un profond bouleversement d'ordre qualitatif et quantitatif. A titre d'exemple, la France est au quatrième rang des pays d'accueil pour les étudiants étrangers. (Tricornot, 2017). C'est pourquoi la mobilité en France s'avère comme une parfaite motivation pour l'apprentissage du français.

En résumant, nous pouvons constater que malgré l'intérêt des enseignants à harmoniser l'apprentissage du français, plusieurs problèmes subsistent entre la formation supérieure et la formation secondaire. Nos observations personnelles nous conduisent à penser que la plupart des difficultés, rencontrées auprès des étudiants en premières années de licence, sont dues à une préparation linguistique insuffisante et mal organisée, ou encore à un manque d'autonomie de l'apprenant qui lui empêche d'accomplir des tâches propres au milieu académique. Par ailleurs, nous sommes convaincus qu'une préparation systématique du projet d'orientation personnel des candidats aux études supérieures serait un bon moyen pour éliminer le choix de parcours aléatoire menant souvent à l'échec. Le rôle appartient également aux universitaires de déterminer des prérequis disciplinaires et de les faire savoir au milieu secondaire, tant aux enseignants qu'aux élèves. Certes, il existe certaines actions d'information de masse, comme par exemple la publicité par médias ou les journées portes ouvertes. Plusieurs organismes opèrent dans le domaine du français, dont la plus ancienne, l'Association slovaque des professeurs de français (SAUF) qui regroupe le plus grand nombre d'enseignants de français à tous les cycles. Ses membres se rencontrent au moins une fois par an à un colloque international pour 
pouvoir se former et échanger avec des collègues slovaques et étrangers. Nous ne pouvons que consentir à l'idée d'Agnesa Fanová qu'il ne sera pas possible de faire face à un recul de l'intérêt au français, s'il n'y a pas d'aide plus active "par l'extérieur et par le haut». Ni les actions attirantes afin de promouvoir la qualité et la quantité de l'enseignement du français, de la part des membres les plus zélés de l'Association qui se battent à tous les fronts pour garder cette langue à l'esprit des Slovaques, ne vont pas suffire (Fanová, 2013).

\section{L'enseignement du français langue appliquée}

La méthodologie en langues étrangères a connu une forte progression dès le début du $\mathrm{XXI}^{\mathrm{e}}$ siècle, mais elle répond toutefois aux critères constants de l'approche communicative. Cette perspective actionnelle "considère avant tout l'usager et l'apprenant d'une langue comme des acteurs sociaux ayant à accomplir des tâches (qui ne sont pas seulement langagières) dans des circonstances et un environnement donnés, à l'intérieur d'un domaine d'action particulier » CECRL : 15).

En répondant aux nouveaux besoins de la société slovaque et aux exigences de la pédagogie des langues étrangères innovatrice, un nouveau programme d'études (en deux niveaux : Licence et Master) intitulé « Langues étrangères et la communication interculturelle » a été accrédité en 2010, et est désormais dispensé au sein de l'Université d'Economie par les enseignants de la nouvelle Faculté des langues appliquée en même année. Ce cursus pluridisciplinaire en LEA s'appuie sur une bonne maîtrise de deux langues étrangères (anglais-allemand et depuis 2012 aussi anglais-français et anglais-espagnol) ainsi que sur l'acquisition des connaissances et techniques dans les matières des sciences économiques. Cette filière basée sur la professionnalisation des étudiants vise le monde des affaires, de l'entreprise et de l'interculturel. La formation en langues appliquées se focalise sur l'exploitation des acquis langagiers et disciplinaires dans la communication correcte et adéquate avec le monde professionnel. Les étudiants sont suscités à apprendre deux langues en faisant leur propre recherche qui comprend par exemple: l'identification des concepts économiques ou géopolitiques essentiels pour remplir des missions de communication et de négociation avec un partenaire étranger; des traductions de textes de spécialité vers la langue maternelle ou vice versa etc. Les étudiants apprennent à identifier et à sélectionner diverses ressources spécialisées pour se documenter aux sujets donnés. Ils sont conduits à savoir identifier, dans une activité professionnelle, des points convergents et divergents entre les aires culturelles des langues visées et de leur langue maternelle. En outre, une démarche plurilingue, basée sur le croisement de deux (ou trois) langues est intégré dans leur éducation langagière tout au long de leur cursus universitaire. Il est à noter que l'apprentissage parallèle de plusieurs langues pourrait devenir un avantage, comme des apprenants débutants en français ont déjà une expérience antérieure de l'apprentissage de l'anglais. D'après les observations des pédagogues tchèques, "les propositions des stratégies basées sur l'appui de l'enseignement/apprentissage du français - première langue étrangère sur les savoirs et savoir-faire acquis au cours de l'enseignement/apprentissage de l'anglais première langue étrangère apportent les premières preuves d'un impact positif » (Fenclová, $2015: 8$ ). Il appartient à l'école d'encourager les jeunes à développer leur capacité d'apprendre à l'aide des expériences d'autres langues et d'autres cultures (Birova - Eliasova, 2014 : 76).

Dans le cadre des langues appliquées, l'enseignement du français a pour but de développer le lexique spécialisé relatif au domaine d'application afin de savoir exploiter les connaissances acquises à un domaine professionnel particulier. Les cours mettent en œuvre les compétences permettant de traiter (en réception et en production) des textes portant sur des thèmes dans les domaines particuliers, en 
mobilisant les stratégies qui paraissent le mieux convenir à l'accomplissement des tâches à effectuer. Le contrôle de ces activités par les interlocuteurs conduit au renforcement des compétences.

Des compétences sont développées et évaluées de préférence à travers des situations concrètes et proches de la vie réelle. Le rôle du contexte dans le processus de communication doit être pris en compte, étant donné que chacun de ses niveaux fixent des limites aux interlocuteurs, déterminent des règles et complètent le code verbal ou non-verbal (Hrivíková, 2012: 78). Par suite, la composante communicative va de pair avec la composante interculturelle, plus particulièrement en FLEA qui prépare ses apprenants aux futures professions des entrepreneurs, manageurs, médiateurs ou traducteurs. La communication interculturelle présente une confrontation pluridimensionnelle avec la civilisation dont les résultats aident à créer des attitudes positives envers les étrangers, à s'affranchir de préjugés ainsi qu'acquérir la capacité d'une critique constructive à propos des faits négatifs dans le pays dont la langue il apprend (Horváthová, $2011: 28$ ).

Chaque discours scientifique ou académique, destiné à un public spécifique, occupe une place importante dans le contexte social et devient une maille du réseau de discours similaires qui tâchent de renouer les liens avec des connaissances du passé pour développer l'état du savoir actuel et futur. Le discours est censé être un produit complexe de l'usage de la langue et des facteurs extralinguistiques, qui est réalisé à l'écrit ou à l'oral. C'est tout texte dans sa profondeur et sa polysémie comprenant un ensemble de connaissances sur le monde, la société, les codes de communication (Štefančík - Dulebová, 2017: 46). Le rôle de l'enseignant est de sensibiliser les apprenants à une prise de conscience des différences culturelles dans l'enseignement. A titre d'exemple, le langage des couleurs si différencié dans diverses langues, donne la preuve de l'importance de l'aspect culturel dans le processus de l'apprentissage d'une langue (Spišiaková, 2016 : 3). Elle affirme qu'afin de maîtriser une langue étrangère, il faut que son apprentissage soit lié à l'étude approfondie de la culture du pays de cette langue. Cet objectif apparaît d'autant plus pertinent comme son développement systématique a manqué au passé dans le contexte slovaque : «Le programme scolaire néglige un regard critique sur le "soi-même ", sur la relativisation de sa propre culture ... les hypothèses de base concernant la compréhension des « autres » et la compréhension entre les peuples en termes de tolérance et de respect de la diversité ne sont pas formulées » (Ciprianová, Bírová, 2016 :140).

\section{Vers un référentiel transversal}

Le Français sur objectifs universitaires (FOU) cherche à donner une nouvelle dimension au français, celle de langue de communication scientifique. Il conduit à " traiter les formes et les procédures et se définit comme la branche du FOS (français sur objectifs spécifiques): le public cible est caractérisé par la pluralité des spécialités dont les contenus sont principalement de type formel et procédural et dont les objectifs se subdivisent selon trois axes: la vie de l'université, la compréhension orale du discours

universitaire et les exigences de l'expression écrite » (Clavreul - Maillard : 39). Loin de demander simple reformulation de connaissances apprises dans des livres, le rôle des enseignants est de stimuler l'intérêt des apprenants "aux ressemblances et différences, soucieux de dégager les règles de fonctionnement de ce système qu'ils sont en train à la fois de découvrir et d'expérimenter. Et cette compétence transversale de "savoir-interpréter» semble être l'une des pistes intéressantes à emprunter pour aider les étudiants à " apprendre à apprendre » et, in fine, s'enrichir en découvrant une autre manière de penser, un autre type de rapport au savoir» (ibid.). Dans cette lignée, les cours sont organisées en fonction des tâches à accomplir, de sorte que les actes de parole, la grammaire, le lexique, l'orthographe deviennent

XLinguae, Volume 11 Issue 1XL, January 2018, ISSN 1337-8384, eISSN 2453-711X 
des moyens langagiers nécessaires au développement des compétences transversales dont notamment la capacité de saisir, comprendre et traiter l'information. Or, l'étudiant apprend à analyser, interpréter ou réutiliser ses connaissances dans de nouveaux contextes. Par ailleurs, observation, analyse et synthèse, généralisation, induction et déduction, jugement et évaluation, ce sont les démarches intellectuelles mises en place et sollicitées régulièrement auprès des étudiants pour qu'elles deviennent progressivement les leurs pour trouver la solution de problèmes nouveaux et inédits (Dupont - Ossandon, 1994 : 32). Au niveau du style, les étudiants doivent s'adapter au discours académique respectant la forme de français soutenue et normée, tant à l'oral qu'à l'écrit.

Les macrocompétences langagières transversales aux disciplines, formulées par JeanMarc Mangiante, permettent d'élaborer un référentiel de compétences langagières pour la formation linguistique des étudiants et la préparation aux études supérieures. L'objectif de la macrocompétence est de réaliser macrotâche au sens du CECR, c'està-dire un projet comportant un ensemble de tâches réalistes à réaliser selon un certain scénario (Mangiante - Parpette, $2014: 157$ ).

La rédaction d'un écrit académique en langue étrangère est une tâche particulièrement exigeante parce qu'elle suppose une bonne orientation dans le sujet traité et une excellente compétence en langue. Il est fort recommandé de commencer par la compréhension de textes de vulgarisation scientifique pour pouvoir passer aux textes scientifiques et demander tout d'abord à produire des textes plus courts à un plan plus simple, pour arriver aux écrits plus longs et structurés. L'analyse de textes argumentatifs devrait être introduite beaucoup plus tard, après l'analyse de textes à caractère informatif ou descriptif. Au niveau linguistique, c'est notamment le vocabulaire pauvre, la syntaxe inadéquate et fautes d'orthographe qui devraient être travaillés davantage, lorsqu'ils apparaissent régulièrement dans les travaux écrits des étudiants de premières années des études universitaires (Sánchez Presa, 2017 : 92).

La catégorisation des écrits universitaires qui s'appuient sur la compréhension orale des cours magistraux constitue ce que l'on pourrait qualifier de «culture universitaire à la française ». "Chaque catégorie répond à un ensemble de consignes d'écriture, à une mobilisation des compétences cognitives particulières de la part des étudiants quand ils préparent leurs examens à partir de leurs notes de cours et quand ils utilisent les documents annexés aux énoncés » (Mangiante, 2012 :156).

Le mémoire de fin d'études constitue un travail de recherche approfondi destiné à explorer une question, non encore traitée ou à systématiser l'étude d'un problème. Il ne peut se contenter de décrire, mais il doit comporter une analyse rigoureuse du sujet traité ainsi qu'une réflexion et une appréciation critique de la question abordée (ibid.). Le mémoire, comme l'écrit académique plus complexe, suscite un grand respect, les étudiants doivent être bien prêts à cette macrotâche. Ceci est possible par une série de productions moins exigeantes, comme par exemple: faire un résumé, un compte rendu, une dissertation et une synthèse de documents (voir le tableau 1 ci-dessous).

Tableau 1. Macrocomptétences appliquées à la production écrite universitaire.

\begin{tabular}{|c|c|c|}
\hline Macrotâches & Macrocompétences & Productions \\
\hline \multirow{5}{*}{$\begin{array}{l}\text { Reproduire, } \\
\text { reconstituer : } \\
\text { - les données } \\
\text { notionnelles du } \\
\text { cours } \\
\text { - les énoncés } \\
\text { démonstratifs }\end{array}$} & identifier les éléments clés & $\begin{array}{l}\text { réponses aux questions de } \\
\text { cours }\end{array}$ \\
\hline & $\begin{array}{l}\text { repérer les structures } \\
\text { définitoires, explicatives et } \\
\text { expositives du cours }\end{array}$ & $\begin{array}{l}\text { définition, description, } \\
\text { démonstration }\end{array}$ \\
\hline & $\begin{array}{l}\text { définir, nommer, classer } \\
\text { objets, notions, phénomènes }\end{array}$ & explication \\
\hline & appliquer, déduire, induire & \\
\hline & répondre aux injonctions & \\
\hline
\end{tabular}




\begin{tabular}{|c|c|c|}
\hline \multirow{4}{*}{$\begin{aligned} & \text { Reformuler : } \\
&-\quad \text { la } \\
& \text { compilation } \\
& \text { des notes de } \\
& \text { cours } \\
&-\quad \text { les notions } \\
& \text { du cours } \\
& \text { présentes } \\
& \text { dans les } \\
& \text { documents } \\
& \text { annexes }\end{aligned}$} & $\begin{array}{l}\text { comparer, exposer des } \\
\text { notions }\end{array}$ & commentaire de texte \\
\hline & $\begin{array}{l}\text { commenter, donner son } \\
\text { point de vue }\end{array}$ & résumé, compte rendu \\
\hline & altérer, modifier & synthèse de documents \\
\hline & mettre en perspective & dissertation \\
\hline \multirow{6}{*}{$\begin{aligned} & \text { Relier, associer : } \\
&-\quad \text { des } \\
& \text { connaissanc } \\
& \text { es } \\
&- \text { des faits } \\
&- \text { des concepts } \\
&- \text { des } \\
& \text { systèmes }\end{aligned}$} & analyser une situation & document professionnel \\
\hline & $\begin{array}{l}\text { situer des faits dans le } \\
\text { temps, l'espace, le champ } \\
\text { disciplinaire }\end{array}$ & étude de cas \\
\hline & argumenter, contester & travail semestriel \\
\hline & interpréter, estimer, mesurer & \\
\hline & innover, créer & \\
\hline & $\begin{array}{l}\text { relier les informations dans } \\
\text { une progression structurée }\end{array}$ & \\
\hline
\end{tabular}

(Source : Adapté d'après Le Français sur objectif universitaire. Grenoble : PUG,

2014. P. 158-159)

La phase précédant la rédaction même du mémoire de licence consiste à préparer un travail semestriel qui devrait contenir toutes les parties d'un texte académique, à savoir un abrégé et des mots-clés, une introduction comprenant la formulation de la problématique, une hypothèse ou des questions de départ, une méthodologie. L'enseignant joue le rôle d'un conseil pour aider l'apprenant à s'orienter lors de sa préparation à la rédaction.

La rédaction d'un texte de ce genre exige une bonne préparation au niveau de la spécification de la problématique, de la sélection des outils lexicaux et grammaticaux, de la composition syntactique, tout en respectant le style adéquat. Son caractère de discours préparé se traduit par le souci de cohérence au niveau global du texte et de progression des idées, et celui de cohésion au niveau local respectant des normes morphologiques et syntaxiques. La précision terminologique et la correction linguistique et orthographique sont ses attributs inévitables.

Loin d'être uniquement une nouvelle démarche méthodologique, le français sur objectif universitaire est devenu également l'objet de recherche scientifique visant le classement "de différents textes selon leurs règles de composition ou celle de types discursifs qui empruntent à la pragmatique les critères d'un classement par les intentions de communication des destinateurs » (Mangiante, 2012 :156).

Le rôle de la langue académique (correspondant au milieu académique à l'université) et la langue scientifique introduites dans le processus pédagogique consiste à analyser des ressources scientifiques et à produire des textes structurés (Seresová, Breveníková, 2017: 12). Rédiger un texte académique n'est pas une tâche facile, même dans sa propre langue. Dans les écoles secondaires slovaques, on ne prête pas assez d'attention à l'écrit académique aux cours de langue maternelle (Kvapil, 2017 : 94), et encore moins en langue étrangère. La constatation d'un déficit de la compétence rhétorique à l'écrit qu'à l'oral dans le contexte universitaire ainsi que l'hypothèse que l'acquisition d'une compétence de communication scientifique cultivée contribue à la réussite dans les études universitaires et à l'intégration sur le 
marché du travail concurrentiel, ont mené un groupe d'enseignants-chercheurs des langues étrangères appliquées (LEA) (anglais, allemand, français et espagnol) a monter un projet scientifique et pédagogique. Ce projet intitulé «Langue académique et scientifique dans le milieu universitaire » est basé sur les approches innovantes et les expériences acquises lors des cours (séminaires) de langue académique. Son but sera de préparer le cadre théorique et méthodologique de l'enseignement de la langue académique à la Faculté des langues appliquées (FAJ) à l'Université d'Economie de Bratislava. Les résultats d'un sondage par enquête réalisé auprès des étudiants et enseignants de la faculté respective témoignent de l'utilité des acquis en langue académique au cours de leurs études à l'université domicile, de même qu'aux universités d'accueil en France pendant la mobilité étudiante. Il est à souligner que les étudiants apprécient l'intention des pédagogues de concevoir des manuels qui offriront des connaissances, des tâches et des textes liés au discours scientifique en toutes les langues étudiées, et qui seront adaptés aux besoins des apprenants slovaques. Les enseignants sondés ont affirmé une amélioration en matière de l'application des conventions relatives au discours académique ainsi que du style des écrits d'étudiants. Ils ont observé même une meilleure qualité d'interprétation et d'argumentation auprès des étudiants. Au contraire, plusieurs défauts perdurent au niveau des savoir-faire linguistiques et à l'orthographe.

A la fin, nous constatons que ce type de projet a d'autres points positifs. Le projet commun réunissant une équipe mixte de 5 langues représentées: anglais, allemand, français, espagnol, slovaque dont deux de famille francophone, permet d'entamer une coopération enrichissante en termes de théorie linguistique, de base textuelle, de cultures et stratégies de communication. De plus, un contenu et des matériels adaptés au public connu ont forte chance de correspondre à leurs compétences et leurs attentes. D'ailleurs, le fait que la culture et la réalité des apprenants slovaques pourront être intégrées dans le cours et les manuels s'avère être un grand avantage.

\section{Les stratégies et les pratiques pour rendre l'enseignement du français plus efficace}

1 Parmi des stratégies fréquentes appartient l'utilisation des matrices de production qui consiste à faire des variations de différents paramètres d'une même situation de discours. Dans la plupart des exercices d'entrainement en écriture, des consignes orientent à accomplir une tâche bien précise, comme par exemple: relever, citer, formuler, énumérer, comparer, définir, trouver des arguments et d'autres.

2 Il existe des activités qui stimulent davantage la créativité et l'autonomie de l'apprenant en lui demandant de trouver lui-même la démarche pour accomplir une tâche plus complexe, par exemple: résumer un texte, faire une synthèse de documents etc.

3 La pédagogie par projet est encore plus exigeante, mais très recommandée pour préparer des apprenants à la rédaction d'un travail semestriel ou d'un mémoire. Elle implique plusieurs phases, comme celle de formuler la problématique, faire la recherche documentaire et sélectionner des ressources de références, élaborer une introduction, développement ou conclusion, placer des citations ou paraphrases, relire et corriger etc. Le projet sollicite l'étudiant à travailler à son rythme en utilisant ses propres méthodes, ce qui favorise son autonomie. De plus, il l'apprend à prendre la responsabilité et se maîtriser.

4. Il faut veiller à ce que les tâches et les activités dont l'application doit conduire aux objectifs déterminés, ressortent du contenu de tout le programme d'études et non seulement d'une seule matière. "Il est sûr qu'aucun programme n'est susceptible de transmettre aux apprenants tous les savoir-faire dont ils pourront avoir besoin par la suite. On peut 
néanmoins les doter d'un outil de développement continu qui leur permettra d'avoir une meilleure compréhension générale d'eux-mêmes en tant qu'apprenant, du processus d'apprentissage, ainsi que des facteurs qui entrent en jeu dans la communication " (Camillieri, 2002: 5). Pour y arriver, tout le corps enseignant devrait développer les capacités productrices chez les étudiants, et cela dans l'ensemble des cours enseignés. Les supports pédagogiques et le corpus de textes adaptés aux besoins concrets, contribuent considérablement à la progression dans l'apprentissage.

5. Evaluer des compétences transversales est plus difficile qu'évaluer des savoirs. La pratique de telle évaluation formative exige le changement de toute la situation d'apprentissage : de ses objectifs, ses pratiques, ses critères d'évaluation etc. (Rizeková, 2015: 42). Bien que les résultats des évaluations universitaires servent notamment à des fins de classification et certification, il est toutefois efficace de partir parfois des travaux des étudiants effectués pour apprendre à partir des erreurs. E. Stradiotová mentionne deux types d'évaluation dont la première est spontanée et basée sur l'impression, et la seconde - intentionnelle, est par contre, préparée et contrôlée (Stradiotová, 2017:38).

6. Pour arriver à rédiger un texte ou à préparer un exposé inspirant et intéressant, il vaut mieux choisir un sujet correspondant aux intérêts et aux capacités de son auteur. La possibilité d'en effectuer une recherche scientifique et d'établir une fiche terminologique augmente la chance de réussir. De surcroît, l'application du texte élaboré dans le monde académique ou professionnel est une priorité. Et le choix d'un sujet spécifique à un pays ou à une culture donnée fournit de bons points de départ pour divers types de recherche comparée.

\section{Conclusion}

Actuellement, les universités offrant une formation linguistique doivent affronter deux grands problèmes : la régression du taux de poursuite des études et le pourcentage d'échec des étudiants du premier cycle. Vu que la transition de l'école secondaire au degré supérieur est une période difficile au niveau de l'adaptation, force est de constater qu'un rapprochement plus étroit de ces deux mondes est impératif. Ce rapprochement se traduit par l'articulation des objectifs et des contenus au sein des programmes d'études ainsi que par l'amélioration du travail de conseillers d'orientation et d'éducation scolaire et professionnelle.

Pour diminuer le risque de l'échec en français au cours des premières années universitaires, dû soit à une mauvaise orientation de bacheliers sous-estimant leur niveau requis dans les langues, soit par le volume considérable de travail exigé par la filière, il est de rigueur de les guider dans la préparation de leur parcours, par un projet professionnel ou académique. Les bacheliers devraient connaître les maquettes des enseignements du cursus pour pouvoir se décider pour l'une des formations : celle en FLE, FOS ou FLEA. Et il s'avère nécessaire de renseigner les bacheliers sur tous les points pertinents de la formation qu'ils veulent entreprendre.

D'ailleurs, il est le temps de rendre plus fonctionnel le cadre d'actions orientées au développement systématique des aptitudes et des compétences des apprenants slovaques en langues pour qu'ils sachent mieux exploiter leurs capacités actuelles et leurs potentiels qui leur permettront de s'intégrer plus aisément dans la vie sociale et professionnelle. Cette stratégie pourrait, en même temps, faire augmenter l'attractivité des filières d'études en langues.

La mise en œuvre d'un nouveau contenu et une nouvelle méthodologie de français sur objectif universitaire prévoit une formation spécifique des enseignants de FLE ou de 
FOS. Nous nous attendons à ce qu'elle soit réalisée par des séminaires spécialisés, des forums ou colloques qui permettront un échange des savoirs et des expériences parmi des chercheurs, pédagogues et didacticiens à l'échelle nationale et internationale. Et non en dernier lieu, nous supposons que tout enseignant-chercheur engagé fasse des efforts pour s'instruire lui-même dans le domaine qui est en plein essor ses dernières années.

Cet article fait partie du projet pédagogique en cours KEGA 029EU-4/2016 «Langue académique et scientifique dans le milieu universitaire ».

\section{Bibliographic references}

BIROVA, J. - CIPRIANOVA, E. 2016. Le français en Slovaquie: developpement de " l'interculturel » dans les programmes scolaires et situation actuelle. Synergies Europe n 11. pp. 133-147.

CAMILLERI, A. 2002. Introduction de l'autonomie de l'apprenant dans la formation des enseignants. Strasbourg : Editions du Conseil de l'Europe. 84 p. Available online: http://portail-du-fle.info/glossaire/autonomieapprenant formationCamillerilivre.pdf.. [Cit. 2018-1-2]. ISBN 92-871-4982-8

CECRL. Cadre europeen commun de reference pour les langues: apprendre, enseigner, evaluer. Unite des Politiques linguistiques, Strasbourg. Available online: www.coe.int/lang-CECR [Cit. 2017-12-20].

CLAVREUL, V. - MAILLARD, N. - DE LA CORTE, N. - ROUSSEAU-GADET, G. et E. Les etudiants etrangers a l'universite et le français sur objectifs universitaires: l'exemple de l'evaluation. pp. 103-121.

DUPONT, P. - OSSADON, M. 1994. La pedagogie universitaire. Paris: Presses universitaires de France. ISBN 2130465455.

FANOVA, A. 2013. Francuzstina v systeme vyucovania cudzich jazykov u nas - 2 . cast. [online]. [Cit. 2018-1-8].

FENCLOVA, M. 2015. Some New Views on Teaching French with Respect to Teaching/ Learning English (Czech Experience) [Quelques perspectives pour une didactique du français apres l'enseignement/ apprentissage de l'anglais (experience tcheque)]. In: XLinguae. Vol. 8, Issue 4, pp. 2-9. ISSN 1337-8384.

HISTORIQUE ET OBJECTIFS. FENCLOVA, Available online: www.fsok.sk/presentation-de-la-ccfs/qui-sommes-nous/historique-et-objectifs [Cit. 2018-1-2].

HORVATHOVA, B. 2011. Rozvoj kompetencii autonomneho studenta vo vyucovani odborneho jazyka pomocou e-learningu. In: XLinguae Vol. 4, Issue 1, pp. 27-33. ISSN 1337-8384.

HOUCHOT, A. - ROBIN, F. 2007. Les livres de competences: Nouveaux outils pour evaluation des acquis. N. 2007-048. Rapport a monsieur le ministre de l'Education nationale.

HRIVIKOVA, T. 2012. Interkulturna komunikacia z hladiska kontextu alternativny pristup ku studiu interkulturnej komunikacie. In: Lingua et vita: vedecky casopis pre vyskum jazykov a interkulturnej komunikacie. ISSN 1338-6743. V. 1, n. 1, pp. 73-80. KOPECKY, P. 2007. Le français en Slovaquie et son train de vie. Ianua. Revista Philologica Romanica. Vol. 7, pp. 73-79. Romania Minor. ISSN 1616-413X. Available online: www http://www.romaniaminor.net/ianua/ [Cit. 2018-1-2].

KVAPIL, R. 2010. 2017. Komunikacia vakademickej sfere so zameranim na pisomny prejav. Bratislava: Ekonom, pp. 93-112. ISBN 978-80-225-4434-4.

MAGIANTE, J.-M. 2012. Le Français sur Objectif Universitaire: de la maitrise linguistique aux competences universitaires. Synergies Algerie, N 15, 2012, pp. 147166. ISSN-e 1958-5160,

MANGIANTE, J.-M. - PARPETTE, CH. 2014. Le français sur objectif universitaire. Grenoble: PUG, p. 252. 
PROFIL de la politique linguistique éducative 2004-2007. Division des Politiques linguistiques. Strasbourg : Conseil de l'Europe Ministere de l'Education nationale de la Republique slovaque. Available online: https://www.coe.int .

RELATION BILATERALES. Available online: https://www.diplomatie.gouv.fr/fr/dossiers-pays/slovaquie/relations-bilaterales/

RIZEKOVA, I. 2015. Position of Transversal Competencies in University Settings [La place des competences transversales dans le milieu universitaire]. In: XLinguae. V. 8, n. 4, pp. 31-45. ISSN 1337-8384

SANCHEZ PRESA, M. 2017. Komunikacia v akademickej sfere so zameranim na pisomny prejav. Bratislava: Ekonom, pp. 75-92. ISBN 978-80-225-4434-4.

SERESOVA, K. - BREVENIKOVA, D. 2017. Komunikacia v akademickej sfere so zameranim na pisomny prejav. Bratislava: Ekonom, pp. 7-43. ISBN 978-80-2254434-4.

SPISIAKOVA, M. 2016. El analisis contrastivo del uso, significado y connotaciones de los colores en la lengua eslovaca y espanola $=$ The contrastive analysis of the use, meaning and connotations of the colors in the Slovak and Spanish language, In: XLinguae. V 9, n. 3, pp. 104-128. ISSN 1337-8384.

STEFANCIK, R. - DULEBOVA, I. 2017. Jazyk a politika. Bratislava : Ekonom, pp.193. ISBN 978-80-225-4414-6.

STRADIOTOVA, E. 2017. Didakticke strategie pri vyucbe cudzich jazykov. Brno: Tribun EU s.r.o. pp. 24-40. ISBN 978-80-263-1344-1.

TILMAN, F. 2006. Definir les competences transversales pour les enseigner. Le GRAIN. [online Consulte 2017-12-23].

TRICORNOT, A. 2017. La France recule à la quatrieme place pour l'accueil des etudiants etrangers. Le Monde. Available online: http://www.lemonde.fr/campus/article/ 2017/01/12.

Words: 7665

Characters: 53054 (29,47 standard pages)

PhDr. Iveta Rizeková, PhD.

Department of Romance and Slavic languages

Faculty of Applied Languages

University of Economics in Bratislava

Dolnozemská cesta 1

85232 Bratislava

Slovakia

iveta.rizekova@euba.sk

XLinguae, Volume 11 Issue 1XL, January 2018, ISSN 1337-8384, eISSN 2453-711X 